\title{
Immediate effect of a motor control exercise target to the neck muscles on upper cervical range of motion and motor control in patients with temporomandibular disorder
}

\author{
Alexandra Daniele de Fontes Coutinho (D) Ana Izabela Sobral de Oliveira-Souza (iD) Lais Ribeiro Sales, Daniella \\ Araújo de Oliveira
}

Universidade Federal de Pernambuco, Recife, Brazil

\section{$凶$}

Daniella Araújo de Oliveira Av. Jorn. Aníbal Fernandes, 173 - Cidade Universitária, Recife, Pernambuco, Brazil, 50740-560 E-mail: sabinodaniellaufpe@ gmail.com

Edited by:

Marcelo Moraes Valença

Keywords:

Temporomandibular joint disorders Range of motion

Facial pain

Exercise

Rotation

Women

\begin{abstract}
Objective

To evaluate whether a single specific motor control training session for the neck flexor and deep extensor muscles improves upper cervical range of motion and neck motor control in patients with temporomandibular disorder (TMD) and compare them to a group without TMD.

Methods

This is a before and after, controlled study. The TMD group included women aged between 18-45 years old, complaining of pain in the orofacial region in the last 6 months and diagnosed with masticatory myofascial pain according to Research Diagnostic Criteria (RDC) MD). The control group included match-controls without TMD. The participants were evaluated to global and upper (Flexion Rotation Test - FRT) neck range of motion (ROM) and to neck motor control (Cranio-Cervical Flexion Test - CCFT). They were treated with a protocol of specific motor control exercises targeted to flexor and extensor neck muscles for 30 minutes. One day after the protocol the patients were reevaluated.

Results

A total of 23 volunteers were evaluated. The TMD group showed immediate improvement in left cervical rotation $(p=0.043)$ and right FRT $(p=0.036)$, while the control group did not show any improvement. There was no difference between the groups before and after treatment in relation to cervical movements. Regarding cervical motor control in both groups, the highest prevalence was of results between 24 and $26 \mathrm{mmHg}$ after treatment, different from before the intervention (20 and $22 \mathrm{mmHg}$ ) in both groups.

\section{Conclusion}

A single session of specific neck motor control training only improved the left cervical rotation and upper right rotation in the TMD group, but not in the control group. There is no difference at the end of treatment between the groups. Volunteers with TMD showed improvement in the pattern of motor control of the neck when compared to volunteers without TMD.
\end{abstract}




\section{Introduction}

Temporomandibular joint (TMJ) is an element of the stomatognathic system formed by several internal and external structures, it is located anteriorly to the external acoustic meatus, inferiorly to the temporal bone and superiorly to the mandible and contains an intra-articular disk within the articular capsule that divides it into superior and inferior. This joint can perform complex movements such as protrusion, retrusion, elevation and excursions (right and left). The functions of mastication, swallowing, phonation, and cervical posture depend heavily on TMJ function, health, and stability to work properly. ${ }^{1}$

Temporomandibular disorder (TMD), in turn, is a collective term for structural and functional disorders involving the TMJ and/or masticatory muscles, head and neck muscles, and contiguous tissue components. ${ }^{2}$ Biological, anatomical, biomechanical, behavioral, environmental, and emotional factors affect the masticatory system, causing the development of signs and symptoms and/or perpetuation of TMD. ${ }^{3}$ Therefore, TMD can be considered a multifactorial disease entity. ${ }^{4}$ It is primarily characterized by pain and restricted jaw movement, with pain being the most common symptom and the most frequent reason for seeking treatment. ${ }^{4}$

The literature points out that patients with TMD have craniocervical changes besides indicating that orofacial pain may be related to changes in the upper region of the cervical spine. ${ }^{5}$ In addition, patients with TMD have self-reported cervical pain, limited cervical range of motion (ROM),, 0 decreased pain threshold to pressure in the scalene anterior, upper trapezius, and sternocleidomastoid (SSTM) muscles. ${ }^{8,9}$ As well as changes in cervical motor control, resulting from a reduced activity of the deep cervical muscles added to a hyperactivity of the superficial cervical muscles (scalene anterior and sternocleidomastoid muscle) and reduced strength and endurance of the extensor and flexor cervical muscles. 9,10

The relationship between TMD and pain and functional changes in the muscles of the cervical spine can be explained by the neuroanatomical mechanism of convergence between trigeminal afferences and three upper cervical nerves. This convergence occurs in an area called the trigeminocervical nucleus. ${ }^{11}$ The caudal trigeminal nucleus ( $V$ cranial nerve) is a collection of neuronal cells (gray matter) in longitudinal arrangement from the bulbar pyramid to the upper 3-4 segments of the spinal cord. ${ }^{12}$ The anatomical convergence of the nociceptive fibers of the trigeminal $(V)$ nerve, especially those of its ophthalmic branch (V1) with those coming from the cervical spinal nerves from $\mathrm{C} 1$ to $\mathrm{C} 3-\mathrm{C} 4$ is the basis of referred pain from the upper cervical region to the head, including its frontal region. ${ }^{12}$

In this context, several studies have taken into consideration the entire cranio-cervico-mandibular complex in clinical decision making. Among the treatments proposed manual therapy has been considered a viable and useful approach for TMD management. ${ }^{13}$ However, a systematic review by Medlicott and Harris ${ }^{14}$ evaluated the effectiveness of physical therapy interventions for patients with TMD and specifically reported the value of a combined approach of active exercises, manual therapies, and relaxation techniques. While a second review on the effectiveness of physical therapy in patients with TMD found that postural training, manual therapies and exercise demonstrated significant benefits. The authors concluded that active, passive, and postural exercises are effective interventions to decrease symptoms associated with TMD. ${ }^{15}$

Exercise has been an effective treatment for people with chronic neck pain, ${ }^{16}$ and various exercises including motor control training 17,18 and resistance training 19,20 in the neck have been shown to relieve pain, probably due to facilitation of the endogenous analgesia pathway by different mechanisms. In addition, exercise may have positive psychological effects, including reduced pain catastrophizing. ${ }^{21}$

The improvement in clinical symptoms coming from neuromuscular changes provided by training goes according to the type of exercise performed. For example, craniocervical flexion exercise, designed to emphasize activation of the deep cervical flexors and minimize activation of the superficial flexors, ${ }^{17,18}$ increases activation of the deep cervical flexors ${ }^{18}$ which are often less activated in patients with neck pain. ${ }^{22}$ In addition, this exercise reduces activation of the sternocleidomastoid muscle, ${ }^{18}$ which is often hyperactive in association with reduced activity of the deep cervical flexors. ${ }^{22,23}$ Enhanced activation of the deep cervical flexor muscles was not achieved with general neck resistance training, ${ }^{17,18}$ despite comparable changes in pain. The deep cervical extensor muscle, cervical semispinalis, may also exhibit reduced activation in people with neck pain. ${ }^{17}$

Therefore, exercises for the neck muscles showed positive results, so this training target to the neck muscles seems to be a beneficial and positive approach to treat patients 
with TMD, since these have similar motor control disorders to those found in patients with chronic neck pain, making the patient more autonomous with their treatment. Thus, the main objective of the present study was to evaluate whether a single specific motor control training target to flexor and deep extensor neck muscles improves upper and global cervical range of motion and motor control of the cervical spine in patients with TMD. It also compares them to a group without the dysfunction.

\section{Methods}

This is a before and after, controlled study that was carried out in the period from August 2018 to August 2019, in the Laboratory of Learning and Motor Control, of the Department of Physical Therapy, of the Federal University of Pernambuco. The present study was approved by the Ethics Committee of the Federal University of Pernambuco, under process number: 2.131.546.

\section{Sample}

Patients in the TMD group were included according to the following inclusion criteria: women aged between 18 and 45 years; complaints of orofacial pain in the last 6 months; diagnosis of myofascial masticatory pain determined according to the criteria established by the Research Diagnostic Criteria (RDC/TMD); complete dentition except for third molars. The control group included women aged between 18 and 45 years old, with no history of complaints in the orofacial region. In both groups we excluded participants with a history of facial and/or cervical trauma, surgical procedures performed on the cervical spine and/or craniofacial segment, neurological disorders, fibromyalgia, chronic systemic diseases, previous TMD treatments performed in the last six months, use of dental prostheses and ongoing orthodontic treatment.

\section{Sample}

The participants were initially submitted to a screening to determine whether they met the inclusion criteria of the study. This was followed by an evaluation that consisted of using the RDC/TMD and three measurement tests: global cervical range of motion (ROM); upper cervical ROM (flexion rotation test - FRT) and the cervical motor control test (cranio-cervical flexion test - CCFT).

\section{Diagnostic criteria for TMD (RDC/TMD)}

Diagnosis was performed with the RDC/TMD, which is a questionnaire reported by several authors as a reliable tool for evaluating myogenic, arthrogenic, and mixed $T M D$, and is widely used as diagnostic criteria in clinical TMD research. ${ }^{24}$ This questionnaire model was based on the biopsychosocial model of pain. This classification system was based on the biopsychosocial model of pain that included an Axis I which is composed of the physical assessment that includes pain assessment, mouth range of motion, presence, or absence of otologic noises and symptoms, and muscle palpation, using reliable and well operationalized diagnostic criteria and an Axis II assessment of psychosocial status and pain-related disability. Through this one can classify TMD into three groups: Muscle diagnoses (Myofascial pain and/or Myofascial pain with limited opening), Disk displacement (Disk displacement with reduction, Disk displacement without reduction, with limited opening and/or Disk displacement without reduction, without limited opening) and arthralgia, arthritis, arthrosis (arthralgia, TMJ osteoarthritis and/or TMJ osteoarthritis).

\section{Global cervical range of motion}

Cervical range of motion was measured using the $C R O M \circledast$ instrument, which consists of an acrylic device attached to the volunteer's head and secured with a Velcro strap. It has two inclinometers attached to the device plus a removable inclinometer that moves due to the presence of a magnetic field placed on the patient's neck. This instrument aims to determine the range of motion of the cervical spine when it moves in the sagittal and frontal planes, performing flexion, extension, tilt to the right and left and rotation to the right and left. ${ }^{25,26}$

The participants were evaluated for cervical extension, flexion, rotation (right and left) and inclination (right and left). The patients were positioned seated in a chair with their feet on the floor. They were instructed to look at their horizon line and perform the movements twice to obtain an average of each movement.

\section{Upper cervical range of motion}

To evaluate the mobility of the upper cervical spine, segments $\mathrm{Cl}$-C2, the FRT was performed with the CROM $\mathbb{R}$ attached to the head of the volunteer. The participants were in dorsal decubitus and the passive movement of cervical flexion was performed, followed by a rotation of the upper cervical spine (right and left). The movement was performed twice for each side so that the average between them could be calculated. This test is used to measure small changes in the amplitude of the individual upper 
cervical segment. When a change of more than $10^{\circ}$ occurs in the measurement, it is considered a minimal important difference. Volunteers with values less than $33^{\circ}$ means that they have upper neck hypomobility. ${ }^{6}$

\section{Cervical motor control}

The CCFT consisted of a motor control exam in which patients perform the cranio-cervical flexion movement in five progressive stages of increasing pressure by $2 \mathrm{mmHg}$ $(22,24,26,28$, and $30 \mathrm{mmHg})$ with a visual feedback pressure device under the base of the occipital bone (Biofeedback Stabilizer Pressure; Chattanooga, Hixson, TN, USA). Participants were instructed to perform the cranio-cervical flexion movement in a slow, controlled manner until they reached the in requested target pressure levels. The participants had to maintain a constant pressure at each target level for a duration of 10 seconds. They performed the sustained contraction twice at each level, with a 1-minute rest period between repetitions to avoid the effects of fatigue. ${ }^{27}$ For the performance of the test, the participants were in a supine position. The last level that the participant successfully achieved the movement was noted by the evaluator.

\section{Treatment protocol}

On the same day of assessment, the participant underwent a motor control training protocol based on the Falla et al. ${ }^{17}$ protocol, with the aid of a pressure device with visual biofeedback. The cervical motor control training consisted of the cranial flexion exercise, which was performed with the volunteer positioned in a relaxed supine position. This exercise encompasses the deep cervical stabilizer, long head, and long neck muscles. ${ }^{22}$ Participants were instructed to perform and maintain positions that were progressively evolved during the execution of the cranio-cervical flexion movement. During the task, participants were guided from feedback from a pressure unit (Biofeedback Stabilizer Pressure; Chattanooga, Hixson, TN, USA), placed posteriorly to the cervical spine under the occipital bone, to monitor the reduction of cervical lordosis, which occurs with the contraction of the long neck muscle.

The training began with the device inflated to a base pressure of $20 \mathrm{mmHg}$ and the participant was asked to perform a head flexion movement (NOD movement, as short "yes" movement), and she should maintain it for 10 seconds with ten repetitions with a 10-second interval between them, this sequence being like a single repetition that lasts 190 seconds, from then on she should progress the exercise during five stages of $2 \mathrm{mmHg}$ each, reaching the maximum pressure of $30 \mathrm{mmHg}$. The participant should perform the contractions slowly and smoothly, not allowing retraction or elevation of the head of the stretcher and avoiding simultaneous contraction of sternocleidomastoid muscle and scalene.

\section{Data collection procedure}

The data collection was done in two days. At the first day there was the evaluation, composed by the CCFT, the global cervical range of motion (CROM®) and upper ROM (FRT) and the intervention that consisted of motor control exercises. 24 hours later the reevaluation was done using the same instruments and tests of the evaluation.

\section{Data analysis procedure}

The data were arranged in mean and confidence intervals. Statistical analysis was performed in SPSS software version 20.2. For the analysis of data distribution and population histogram, the Shapiro-Wilk normality test was performed to verify the data distribution. For the intragroup comparison (pre- and post-treatment) and for the intergroup comparison, the Student t-test was used, with the p-value set at 0.05 . For the comparison of the prevalence of the motor control test result, the subgroups of $20-22 \mathrm{mmHg}$; 24-26 mmHg; $28-30 \mathrm{mmHg}$ were established, and the comparison before and after treatment within and between group was performed with the chi-square test.

\section{Results}

In all, 23 volunteers were evaluated in the two groups, with no statistical difference between groups in the variables age $(p=0.098)$ and BMI $(p=0.477)$. Patients in the TMD group had a mean age of 27.9 (SD 7.7) years old with a BMI of 22.5 (SD 3.8), while in the control group the mean age was 22.3 (SD 1.6) years old and BMl of 21.1 (SD 4.7).

The TMD group showed immediate improvement in left rotation $(p=0.043)$ and right FRT $(p=0.036)$ after the application of the protocol, while the control group did not show any improvement (Tables 1 and 2). However, there was no difference between the groups before and after treatment regarding cervical movements (Tables 3 and 4).

Regarding neck motor control in both TMD and control groups, the highest prevalence was between 24 and $26 \mathrm{mmHg}$ at the end of treatment, unlike before the intervention, when the highest prevalence was between 20 and $22 \mathrm{mmHg}$ in the TMD and control group (Figures 1 and 2). 
Table 1. Comparison of before and after TMD patients' cervical range of motion variables

\begin{tabular}{cccccc}
\hline Variables & Before & After & MD (95\%Cl) & pValue \\
Flexion & $47.1(10.5)$ & $44.4(11.1)$ & $2.8(-2.5: 8)$ & 0.279 \\
Extension & $52.4(8.2)$ & $49.9(8.2)$ & $2.5(-1.25: 6.3)$ & 0.176 \\
Right lateral flexion & $34.9(6.5)$ & $36.2(6.8)$ & $-1.3(-4.5: 1.8)$ & 0.377 \\
Left lateral flexion & $37.7(6.7)$ & $37.3(5.6)$ & $0.3(-1.5: 2.2)$ & 0.697 \\
Right rotation & $60.4(6.7)$ & $62.9(6.6)$ & $-2.5(-5.9: 0.9)$ & $3.7(0.1: 7.3)$ & 0.134 \\
Left rotation & $63.0(7.2)$ & $59.3(8.5)$ & $-2.8(-5.4:-0.2)$ & $0.043^{*}$ \\
Right FRT & $36.8(11.4)$ & $39.6(8.5)$ & $-2.6(-7: 1.8)$ & $0.036^{*}$ \\
Left FRT & $38(10.9)$ & $40.6(11.8)$ & & 0.232
\end{tabular}

MD: mean differences; FRT: flexion rotation test.

Table 2. Comparison between TMD and control groups, in cervical range of motion variables

\begin{tabular}{cccccc}
\hline Variable & TMD & Control & MD (95\%Cl) & pValue \\
Flexion & $48.0(5.6)$ & $48.0(2.3)$ & $0(-6.4: 6.4)$ & 1.000 & 0.351 \\
Extension & $56.2(6.4)$ & $56.2(6.4)$ & $-3.3(-11.7: 5)$ & 0.137 \\
Right lateral flexion & $39.2(5)$ & $40.7(5.8)$ & $-1.5(-3.7: 0.7)$ & 0.415 \\
Left lateral flexion & $40.5(4.3)$ & $42.0(4.4)$ & $-1.5(-5.8: 2.8)$ & 0.593 \\
Right rotation & $57.5(6.6)$ & $59.2(8.7)$ & $-1.7(-9.2: 5.8)$ & 0.477 \\
Left rotation & $59.2(8.7)$ & $62.2(5)$ & $-2.5(-10.9: 5.9)$ & $-2.2(-8: 3.6)$ & 0.381 \\
Right FRT & $38.2(5)$ & $40.0(2)$ & $-3.3(-9.8: 3.1)$ & 0.240 \\
\hline Left FRT & $37.2(6)$ & $40.5(1.8)$ & &
\end{tabular}

MD: mean differences; FRT: flexion rotation test.

Table 3. Comparison between the TMD patients and the control group in the variables of cervical range of motion, before the intervention.

\begin{tabular}{cc|c|c|c|}
\hline Variable & TMD & Control & MD (95\%Cl) & PValue \\
Flexion & $47.1(10.5)$ & $48.0(5.7)$ & $-0.9(-10.2: 8.6)$ & 0.848 \\
Extension & $52.4(8.2)$ & $52.8(2.3)$ & $-0.4(-8.7 ; 7.9)$ & 0.917 \\
Right lateral flexion & $34.9(6.5)$ & $39.2(5)$ & $-4.3(-10.4: 1.8)$ & 0.158 \\
Left lateral flexion & $37.5(5.6)$ & $40.5(4.3)$ & $-2.8(-8.8 ; 3.2)$ & 0.348 \\
Right rotation & $60.4(6.7)$ & $57.5(6.7)$ & $2.9(-3.7: 9.5)$ & 0.372 \\
Left rotation & $63.1(7.2)$ & $59.6(8.7)$ & $3.4(-4.1: 10.0)$ & 0.358 \\
Right FRT & $36.8(11.4)$ & $38.2(5)$ & $-1.4(-11.5: 8.7)$ & 0.776 \\
Left FRT & $38.0(11)$ & $37.2(6)$ & $0.8(-8.9: 10.6)$ & 0.861 \\
\hline
\end{tabular}

MD: mean differences; FRT: flexion rotation test.

Table 4. Comparison between the TMD patients and the control group in the variables of cervical range of motion, after the intervention.

\begin{tabular}{c|c|c|c|c}
\hline Variable & TMD & Control & MD (95\%Cl) & pValue \\
Flexion & $44.3(11.1)$ & $48(2.3)$ & $-3.6(-13.3: 6)$ & 0.439 \\
Extension & $49.9(8.2)$ & $56.2(6.4)$ & $-6.3(-14: 1.5)$ & 0.106 \\
Right lateral flexion & $36.2(6.8)$ & $40.7(5.8)$ & $-4.4(-10.9: 2.1)$ & 0.172 \\
Left lateral flexion & $37.3(5.6)$ & $42.0(4.4)$ & $-4.6(-9.9: 0.62)$ & 0.081 \\
Right rotation & $62.9(6.6)$ & $59.2(8.7)$ & $3.7(-3.3: 10.8)$ & 0.280 \\
Left rotation & $59.3(8.5)$ & $62.2(5)$ & $-2.8(-8.8: 3.2)$ & 0.343 \\
Right FRT & $39.6(8.5)$ & $40.0(2)$ & $-0.75(-8.1: 6.6)$ & 0.836 \\
Left FRT & $40.6(11.8)$ & $40.5(1.8)$ & $0.09(-10.2: 10.3)$ & 0.986 \\
\hline
\end{tabular}

MD: mean differences; FRT: flexion rotation test. 


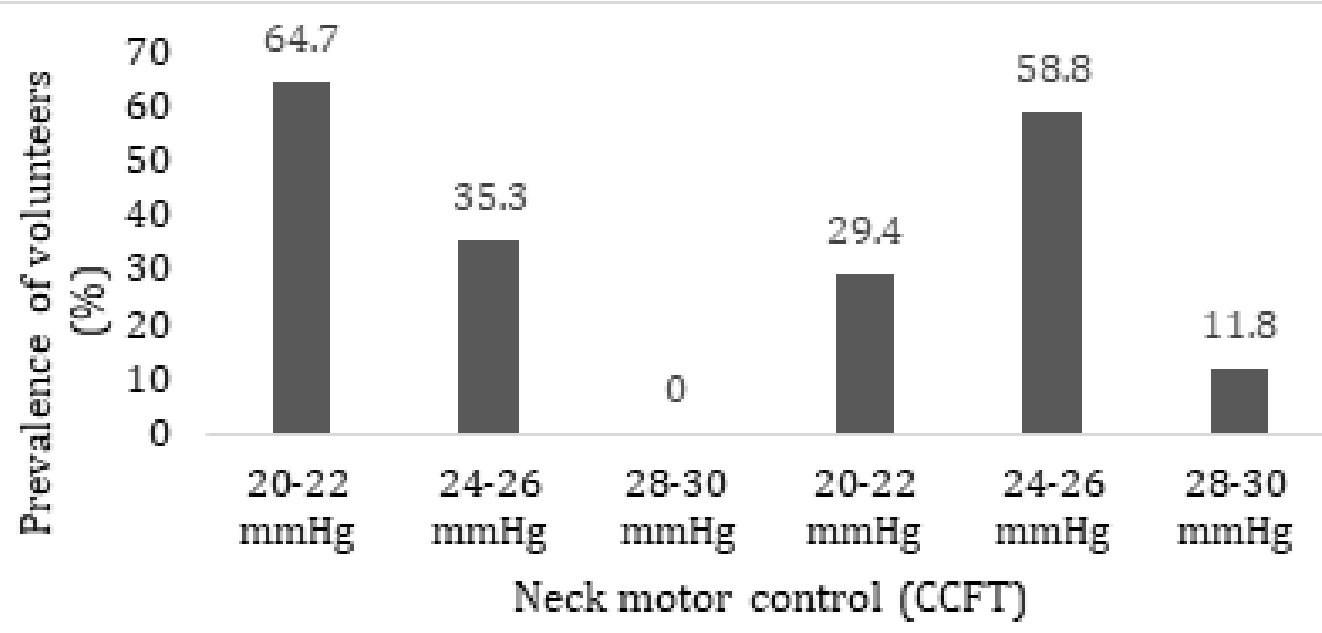

Figure 1. Comparison of prevalence of volunteers with TMD in the cervical motor control test.

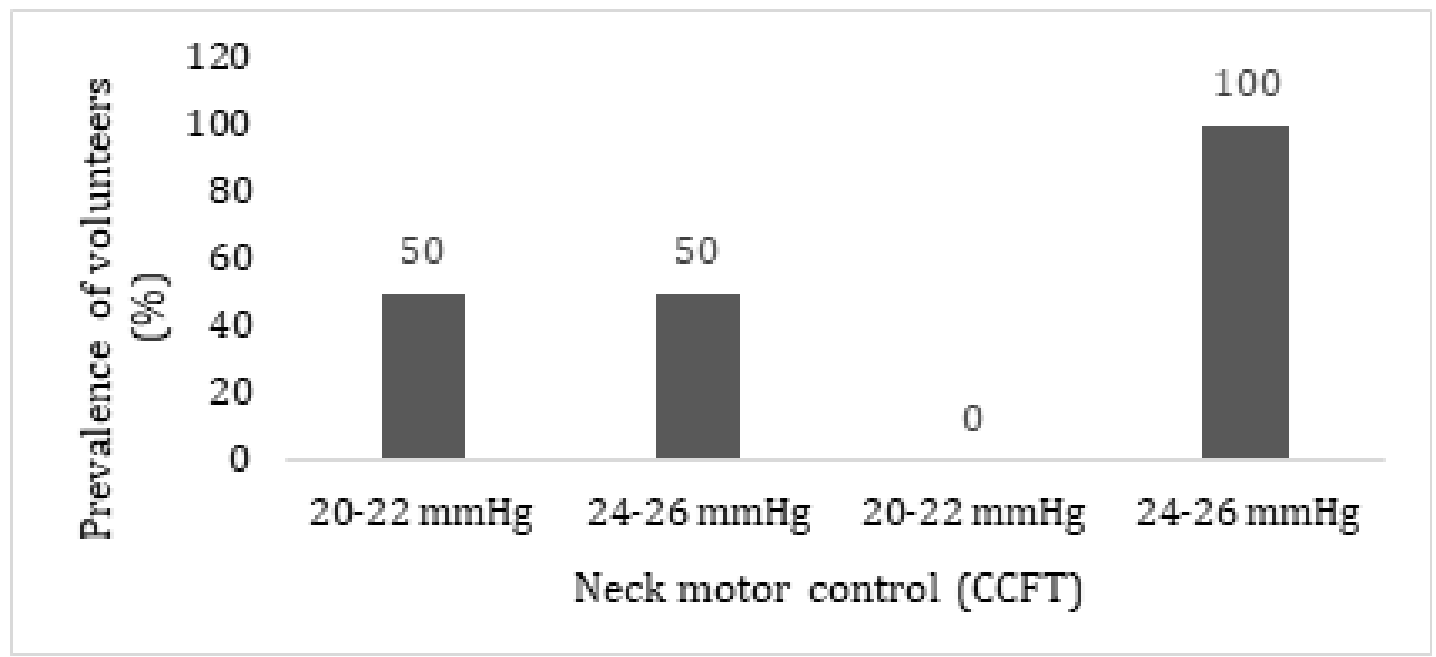

Figure 2. Comparison of prevalence of volunteers without the dysfunction on the cervical motor control test. 


\section{Discussion}

These results indicate that the cervical motor control protocol seems to be an interesting approach strategy to patients with TMD, since only one day of intervention has showed changes in neck mobility and neck motor control. However, these results were not seen when the protocol was applied to volunteers without neck pain.

The different results found between the groups could be due to the fact that healthy volunteers do not present changes in mobility level and cervical motor control as it commonly happens in patients with TMD. ${ }^{11}$ Thus, it is not possible to verify any biomechanical changes in one day of protocol in people who do not present previous neck biomechanical alterations.

The protocol emphasizes and confirms that the neck biomechanical changes present in patients with TMD are due especially to the motor and movement control difficulties that these patients have. Therefore, training and practice in the correct execution of movements will result in clinical improvement in this group of patients. ${ }^{9}$ Shimada et al. ${ }^{28}$ found in a previous systematic review that studies who applied exercise therapy for the treatment of TMD showed positive effects on various clinical conditions of TMD including pain and disability. The study by GarrigósPedrón et al. ${ }^{29}$ concluded that after performing physical therapy treatment, such as cervical manual therapy in chronic cervicalgia and cervical and orofacial treatment in $T M D$, there was a reduction in pain intensity.

However, it is known that association between exercise therapy and manual therapy could be more beneficial to patients with chronic pain compared to the application of each technique isolate. Previous studies have demonstrated that the inclusion of manual therapies in the TMJ, cervical, and masticatory regions was able to induce clinical symptoms (i.e., tinnitus-related disability, TMD-related disability), psychological (i.e., depressive symptoms), and physical (i.e., active mandibular range of motion) improvements in patients with TMD. ${ }^{30}$

Despite the neck motor control protocol has been seen as a very promising treatment approach to patients with TMD, since it makes the patients more autonomous with their treatment, probably an ideal number of sessions would be necessary, since our propose immediate effects was not effective for all patients. ${ }^{17}$

\section{Conclusions}

A single specific motor control training for the flexor and deep extensor muscles of the cervical spine only improves neck range of motion of left and superior right rotation in the TMD group, but not in the control group. There is no difference at the end of treatment between the groups. Regarding motor control of the cervical spine, patients with TMD benefited from the protocol by improving their motor control pattern, while this did not happen in the control group.

Conflict of Interest: The authors declare no conflict of interest.

\section{Funding information}

Coordenação de Aperfeiçoamento de Pessoal de Nível Superior, Grant/Award Number: 001

Alexandra Daniele de Fontes Coutinho

https://orcid.org/0000-0001-9287-9320

Ana Izabela Sobral de Oliveira-Souza

https://orcid.org/0000-0002-7825-2676

Lais Ribeiro Sales

https://orcid.org/

Daniella Araújo de Oliveira

https://orcid.org/0000-0002-6013-978X

\section{References}

1. Pereira KNF, de Andrade LLS, da Costa MLG and Portal TF. Sinais e sintomas de pacientes com disfunção temporomandibular. Rev CEFAC 2005;7(2):221-228

2. Okeson JP and de Kanter R. Temporomandibular disorders in the medical practice. I Fam Pract 1996;43(4):347-356

3. Tuncer $A B$, Ergun $N$, Tuncer $A H$ and Karahan $S$. Effectiveness of manual therapy and home physical therapy in patients with temporomandibular disorders: A randomized controlled trial. J Bodyw Mov Ther 2013;17(3):302-308 Doi:10.1016/j. jbmt.2012.10.006

4. Leeuw R and Klasser GD. Orofacial pain: guidelines for assessment, diagnosis, and management. Am J Orthod Dentofacial Orthop 2008;134(1):171 Doi:10.1016/i. ajodo.2008.05.001

5. Milanesi JDM, Corrêa ECR, Borin GS, Souza JA and Pasinato F. Atividade elétrica dos músculos cervicais e amplitude de movimento da coluna cervical em indivíduos com e sem DTM. Fisioter e Pesq 2011; 18(4):317-322 Doi:10.1590/s1809-29502011000400004

6. Ogince $M$, Hall T, Robinson K and Blackmore 
AM. The diagnostic validity of the cervical flexionrotation test in $\mathrm{Cl} / 2$-related cervicogenic headache. Man Ther 2007;12(3):256-262 Doi:10.1016/i. math.2006.06.016

7. Soares JC, Weber P, Trevisan ME, Trevisan CM and Rossi AG. Correlação entre postura da cabeça, intensidade da dor e índice de incapacidade cervical em mulheres com queixa de dor cervical. Fisioter Pesq 2012;19(1):68-72 Doi:10.1590/S1809-29502012000100013

8. Costa DR, Lima Ferreira AP, Pereira TA, Porporatti AL, Conti PC, Costa YM and Bonjardim LR. Neck disability is associated with masticatory my ofascial pain and regional muscle sensitivity. Arch Oral Biol 2015;60(5):745-752 Doi:10.1016/i.archoralbio.2015.02.009

9. Menezes Kinote APB, Monteiro LT, Vieira AAC, Ferreira NMN and Vasconcellos Abdon APJ. Perfil funcional de pacientes com disfunção temporomandibular em tratamento fisioterápico. Rev Bras Promoç Saúde 201 1;24(4):306-312 Doi:10.5020/2087

10. Armijo-Olivo $S$ and Magee D. Cervical musculoskeletal impairments and temporomandibular disorders. J Oral Maxillofac Res 2013;3(4):e4 Doi:10.5037/ jomr.2012.3404

11. Kraus S. Temporomandibular disorders, head and orofacial pain: cervical spine considerations. Dent Clin North Am 2007;51(1):161-193, vii Doi:10.1016/i. cden.2006.10.001

12. Bogduk N. The neck and headaches. Neurol Clin 2004;22(1):151-171, vii Doi:10.1016/s07338619(03)00100-2

13. Armijo-Olivo S, Pitance L, Singh V, Neto F, Thie N and Michelotti A. Effectiveness of Manual Therapy and Therapeutic Exercise for Temporomandibular Disorders: Systematic Review and Meta-Analysis. Phys Ther 2016;96(1):9-25 Doi:10.2522/ptj.20140548

14. Medlicott MS and Harris SR. A systematic review of the effectiveness of exercise, manual therapy, electrotherapy, relaxation training, and biofeedback in the management of temporomandibular disorder. Phys Ther 2006;86(7):955-973

15. McNeely ML, Armijo Olivo S and Magee DJ. A systematic review of the effectiveness of physical therapy interventions for temporomandibular disorders. Phys Ther 2006;86(5):710-725

16. Miller J, Gross A, D'Sylva J, Burnie SJ, Goldsmith $\mathrm{CH}$, Graham N, . . . Hoving JL. Manual therapy and exercise for neck pain: A systematic review. Man Ther 2010;Doi:10.1016/i.math.2010.02.007

17. Falla D, Lindstrøm R, Rechter L, Boudreau $S$ and Petzke F. Effectiveness of an 8-week exercise programme on pain and specificity of neck muscle activity in patients with chronic neck pain: a randomized controlled study. Eur
J Pain 2013;17(10):1517-1528 Doi:10.1002/j.15322149.2013.00321.x

18. Jull GA, Falla D, Vicenzino B and Hodges PW. The effect of therapeutic exercise on activation of the deep cervical flexor muscles in people with chronic neck pain. Man Ther 2009;14(6):696-701 Doi:10.1016/i. math.2009.05.004

19. Bronfort G, Evans R, Nelson B, Aker PD, Goldsmith CH and Vernon $\mathrm{H}$. A randomized clinical trial of exercise and spinal manipulation for patients with chronic neck pain. Spine (Phila Pa 1976) 2001;26(7):788-797 Doi: 10.1097/00007632-200104010-00020

20. Ylinen J, Takala EP, Nykänen M, Häkkinen A, Mälkiä $E$, Pohjolainen $T, \ldots$ Airaksinen $O$. Active neck muscle training in the treatment of chronic neck pain in women: a randomized controlled trial. Jama 2003;289(19):25092516 Doi:10.1001/jama.289.19.2509

21. Slepian P, Bernier E, Scott W, Niederstrasser NG, Wideman $T$ and Sullivan $M$. Changes in pain catastrophizing following physical therapy for musculoskeletal injury: the influence of depressive and post-traumatic stress symptoms. I Occup Rehabil 2014;24(1):22-31 Doi:10.1007/s10926-013-9432-2

22. Falla D, Jull G, Dall'Alba P, Rainoldi A and Merletti R. An electromyographic analysis of the deep cervical flexor muscles in performance of craniocervical flexion. Phys Ther 2003;83(10):899-906

23. O'Leary S, Cagnie B, Reeve A, Jull G and Elliott JM. Is there altered activity of the extensor muscles in chronic mechanical neck pain? A functional magnetic resonance imaging study. Arch Phys Med Rehabil 2011 ;92(6):929934 Doi:10.1016/i.apmr.2010.12.021

24. Schiffman E, Ohrbach R, Truelove E, Look J, Anderson G, Goulet JP, . . . Dworkin SF. Diagnostic Criteria for Temporomandibular Disorders (DC/TMD) for Clinical and Research Applications: recommendations of the International RDC/TMD Consortium Network and Orofacial Pain Special Interest Groupt. J Oral Facial Pain Headache 2014;28(1):6-27 Doi:10.11607/jop. 1151

25. Audette I, Dumas JP, Côté JN and De Serres SJ. Validity and between-day reliability of the cervical range of motion (CROM) device. I Orthop Sports Phys Ther 2010;40(5):318-323 Doi:10.2519/jospt.2010.3180

26. Oliveira-Souza AIS, Florencio LL, Carvalho GF, Fernández-De-Las-Peñas $C$, Dach $F$ and Bevilaqua-Grossi $D$. Reduced flexion rotation test in women with chronic and episodic migraine. Braz J Phys Ther 2019;23(5):387394 Doi:10.1016/i.bipt.2019.01.001

27. Armijo-Olivo S, Silvestre R, Fuentes J, da Costa BR, Gadotti IC, Warren S, ... Magee DJ. Electromyographic activity of the cervical flexor muscles in patients with temporomandibular disorders while performing the 
craniocervical flexion test: a cross-sectional study. Phys Ther 2011;91(8):1184-1197 Doi:10.2522/ pti. 20100233

28. Shimada A, Ishigaki S, Matsuka Y, Komiyama O, Torisu $T$, Oono $Y, \ldots$ Sasaki K. Effects of exercise therapy on painful temporomandibular disorders. J Oral Rehabil 2019;46(5):475-481 Doi:10.1111/joor. 12770

29. Garrigós-Pedrón M, La Touche R, Navarro-Desentre $P$, Gracia-Naya M and Segura-Ortí E. Effects of a Physical Therapy Protocol in Patients with Chronic Migraine and T emporomandibular Disorders: A Randomized, SingleBlinded, Clinical Trial. J Oral Facial Pain Headache 2018;32(2):137-150 Doi:10.11607/ofph. 1912

30. Delgado de la Serna P, Plaza-Manzano G, Cleland J, Fernández-de-Las-Peñas $C$, Martín-Casas $P$ and Díaz-Arribas M. Effects of Cervico-Mandibular Manual Therapy in Patients with Temporomandibular Pain Disorders and Associated Somatic Tinnitus: A Randomized Clinical Trial. Pain Med 2020;21(3):613624 Doi: $10.1093 / \mathrm{pm} / \mathrm{pnz} 278$ 